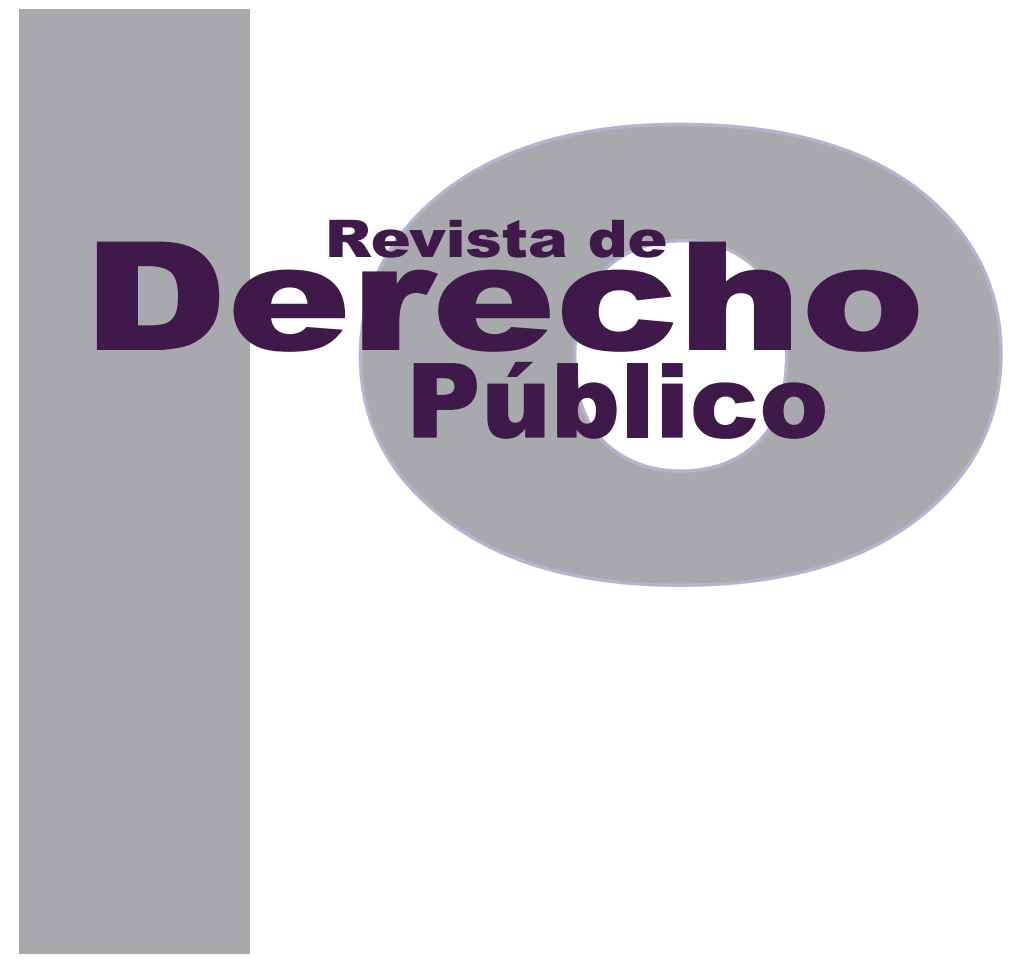

\title{
LA INTERACCIÓN ENTRE EL COMERCIO Y EL MEDIO AMBIENTE EN LOS MARCOS MULTILATERAL, REGIONAL Y LOCAL
}

\author{
Víctor Alonso Flórez VARgas \\ Documento de reflexión no derivado de investigación \\ DOI: http://dx.doi.org/10.15425/redepub.34.2015.20 \\ Universidad de los Andes \\ Facultad de Derecho \\ Revista de Derecho Público N. ${ }^{\circ} 34$ \\ Enero - Junio de 2015. ISSN 1909-7778
}




\title{
La interacción entre el comercio y el medio ambiente en los marcos multilateral, regional y local
}

\section{Resumen}

Se analizarán los distintos instrumentos y escenarios a nivel multilateral, regional y local, donde hay lugar a la interacción comercio/medio ambiente, con el objetivo de determinar el alcance y las posibilidades efectivas para los países de contribuir a la mitigación del daño ambiental a través de ellos. Se hará un estudio cualitativo y de nivel descriptivo sobre la viabilidad práctica de las herramientas jurídicas contenidas en tratados tales como los acuerdos multilaterales sobre medio ambiente (амима), el Protocolo de Kioto, el Acuerdo de Marrakech, la Declaración de Doha, entre otros. Se demostrará que la interacción comercio/medio ambiente varía según se esté en un escenario multilateral, regional o local, comercial o no comercial, en la Unión Europea (UE) o en Estados Unidos; así mismo, que existen tanto amenazas como oportunidades para los países, los mercados locales y las empresas, en las que el desafío para todos consiste en aprovechar las oportunidades y reducir las amenazas.

Palabras clave: tratados ambientales, comercio internacional, multilateralismo, cambio climático.

\section{Interaction between trade and environment in multilateral, regional and local frames}

\begin{abstract}
In this document, there will analyzed the various instruments and scenarios in multilateral, regional and local levels, that give place to the trade / environment interaction, in order to determine the effective opportunities for countries to contribute, through them, to the mitigation of environmental damage. There will be implemented a qualitative and descriptive study on the practical feasibility of legal tools contained in international agreements such as AMUMAs, the Kyoto Protocol, the Marrakech Agreement, etc. It will be demonstrated that the trade/environment interaction can vary, according to the different scenarios such as the multilateral, regional or local, commercial or non-commercial ones, as well as the European Union or in the United States. Likewise, the prove of existence of both, threats and opportunities for countries and local markets and companies, where the challenge consists of taking advantage of the opportunities given and reducing threats.
\end{abstract}

Keywords: environmental agreements, international trade, multilateralism, climate change.

\section{A interação entre o comércio e o meio ambiente nos contextos multilateral, regional e local Resumo}

Serão analisados os distintos instrumentos e cenários a nível multilateral, regional e local, onde há lugar à interação comércio/meio ambiente, com o objetivo de determinar o alcance e as possibilidades efetivas para os países de contribuir com a mitigação do dano ambiental através deles. Será feito um estudo qualitativo e de nível descritivo sobre a viabilidade prática das ferramentas jurídicas contidas em tratados tais como os acordos multilaterais sobre meio ambiente (Амима), o Protocolo de Kioto, o Acordo de Marrakech, a Declaração de Doha, entre outros. Será demonstrado que a interação comércio/meio ambiente varia segundo o cenário multilateral, regional ou local, comercial ou não comercial, na União Europeia (UE) ou nos Estados Unidos; também, que existem tanto ameaças como oportunidades para os países, os mercados locais e as empresas, nas que o desafio para todos consiste em aproveitar as oportunidades e reduzir as ameaças.

Palavras-chave: tratados ambientais, comércio internacional, multilateralismo, mudança climática. 


\title{
La interacción entre el comercio y el medio ambiente en los marcos multilateral, regional y local*
}

\author{
Víctor Alonso Flórez Vargas**
}

\section{SUMARIO}

Introducción - I. EL MEDIO AMBIENTE EN EL MULTILATERALISMO - A. La Cumbre de Río - B. El Protocolo de Kioto y los mercados de carbono - C. Los AMUMA - II. EL MEDIO AMBIENTE EN EL COMERCIO - A. La OMC y el cCMA - 1. El GATT - 2. El Acuerdo sobre Obstáculos Técnicos al Comercio - 3. El Acuerdo sobre la Aplicación de Medidas Sanitarias y Fitosanitarias - B. Conferencias ministeriales y la Declaración de Doha - III.EL MEDIO AMBIENTE EN EL COMERCIO REGIONAL Y BILATERAL - A. La experiencia de la Unión Europea - B. El caso TLCAN - C. América Latina frente a las iniciativas ambientales de la Unión Europea y los Estados Unidos - D. El caso Mercosur - E. El caso de un acuerdo bilateral en Latinoamérica - IV. CONCLUSIONES - Referencias.

\footnotetext{
* Cómo citar este artículo: Flórez Vargas, V. A. (Junio, 2015). La interacción entre el comercio y el medio ambiente en los marcos multilateral, regional y local. Revista de Derecho Público, 34. Universidad de los Andes (Colombia).

** Abogado, Universidad Industrial de Santander; candidato a magister en Propiedad Intelectual, de la Facultad Latinoamericana de Ciencias Sociales (FLACSO), sede Argentina.
} 
Introducción

En el presente documento se pretende hacer un análisis de la relación comercio y medio ambiente en el marco de la liberalización económica mundial, donde se han transformado las condiciones, modos, dinámicas y volúmenes de las economías domésticas (de cara a dicha liberalización comercial) y de la actividad económica global en general. El medio ambiente es un correlato obligatorio de estos sucesos comerciales, cuando versan, en últimas, sobre materia prima constituida por recursos naturales (energía, petróleo, agua, vegetales, animales, aire, minerales, entre otros) que, siendo parte de un ecosistema específico, ocupan un lugar fundamental en el medio ambiente como un todo; donde cualquier cambio cualitativo o cuantitativo significativo de aquellos puede comprometer, positiva o negativamente, la calidad y sostenibilidad de este.

El análisis, inicialmente, tiene por bien establecer el marco multilateral en el que el tema del medio ambiente se desenvuelve: tanto aquel ligado directamente al comercio (marco Organización Mundial del Comercio -omc-) como al que no (conferencias de Naciones Unidas sobre el medio ambiente y el desarrollo y los acuerdos multilaterales sobre el medio ambiente -AmuMA-, fundamentalmente). La intención es indagar sobre el tratamiento que se le ha dado a la interacción comercio/medio ambiente desde el multilateralismo, revisando no solo los contenidos sustanciosos de los distintos instrumentos internacionales que lo sustentan, sino también, y de ser posible, los resultados o efectos de di- cho tratamiento en la realidad ambiental global que, en últimas, es el objetivo esencial de tales instrumentos.

Se abordarán, además, algunas experiencias regionales y nacionales, tales como: la Comunidad Europea, los acuerdos regionales del Tratado de Libre Comercio de América del Norte (TLCAN), el Mercado Común del Sur (Mercosur) y los acuerdos de libre comercio que los Estados Unidos han firmado con algunos países de Latinoamérica. A la par del estudio de los marcos jurídicos internacionales, se expondrán algunos comentarios elaborados por autores e institutos investigadores de la relación comercio/medio ambiente, en el punto de los beneficios o perjuicios que ha traído o, potencialmente, puede traer para cada ámbito dicha interacción.

\section{EL MEDIO AMBIENTE EN EL MULTILATERALISMO}

En el marco multilateral el asunto del medio ambiente se inaugura en 1972 con la Conferencia de Naciones Unidas sobre el Medio Humano, en Estocolmo, Suecia. Esta conferencia estableció el Programa de las Naciones Unidas para el Medio Ambiente (PNUMA), el cual, si bien se centraba en el medio ambiente, no le daba la debida relevancia al tema del desarrollo (PNUMA, 2001). Es así como, Naciones Unidas en 1985 crea la Comisión Mundial sobre el Medio Ambiente y el Desarrollo. Esta comisión expide en agosto de 1987 el famoso Informe Brundtland, titulado "Nuestro Futuro Común", para el cual el tratamiento al problema del medio 
ambiente debe entronizarse en el concepto de "desarrollo sustentable", entendido como aquel que "satisface las necesidades del presente sin comprometer la capacidad de las futuras generaciones de satisfacer las suyas" (Naciones Unidas, 1987, p. 15).

\section{A. La Cumbre de Río}

En 1992 se celebra la Conferencia de las Naciones Unidas sobre el Medio Ambiente y el Desarrollo en Río de Janeiro (Cumbre de Río). Dentro de los principales logros y alcances de la conferencia están, por un lado, el conjunto de principios sobre medio ambiente y desarrollo, condensados en la Declaración de Río y, por el otro, importantes documentos como la Agenda 21, la Convención sobre la Diversidad Biológica (CDB), la Declaración sobre los Bosques y las Masas Forestales, la Convención Marco de Naciones Unidas sobre el Cambio Climático, entre otros.

La Cumbre de Río aparece en un contexto que reconoce la existencia de graves y diversos problemas relacionados con el medio ambiente y el desarrollo como: la contaminación atmosférica, el inicio del agotamiento de los recursos naturales renovables (peces y bosques) y no renovables (petróleo, carbón), la sobredemanda de energía, la pobreza en la mayor parte del globo, las crisis alimentarias y la desigualdad en la distribución de la riqueza. Problemas que atañen a los países desarrollados (PD), los países en desarrollo (PED) y los países menos desarrollados (PMD), en circunstancias y responsabilidades específicas para cada uno, de acuerdo con sus condiciones, especialmente, económicas y am- bientales. De cara a estas problemáticas es que se plantean los principios de la Declaratoria de Río, como marco rector de los esfuerzos políticos, sociales y económicos que han emprendido todos los países para su solución.

Los principios ubican como beneficiarios del desarrollo sustentable a los seres humanos (principio 1), y reconocen que los países son soberanos respecto del aprovechamiento de los propios recursos que posean (principio 2), siempre y cuando no afecten al medio ambiente y lo hagan en el contexto, precisamente, del desarrollo sustentable, teniendo en cuenta las generaciones futuras (principios 3 y 4). Y, siendo los seres humanos los principales beneficiarios, se hace indispensable erradicar la pobreza del planeta, tal y como reza el principio 5 de la Declaratoria.

Así mismo, en el principio 7 se insta a todos los Estados a comprometerse en la conservación, protección y restablecimiento del ecosistema mundial, teniendo en cuenta que han contribuido en mayor o menor medida a la degradación del medio ambiente (cláusula de las responsabilidades comunes pero diferenciadas). Esta responsabilidad común pero diferenciada tiene que ver, por un lado, con el nivel de aporte que ha hecho cada país a la contaminación del ambiente (los PD son quienes más han contaminado) y, por el otro, con los recursos disponibles y las capacidades que cada uno tiene para responder en los procesos de mitigación (los países en desarrollo - PED) y poco desarrollados son los que, en principio, no disponen de recursos y no ostentan capacidades). 
Dicha responsabilidad también se evidencia cuando en el principio 16 se pide a los Estados la internalización de los costos ambientales, de modo que quien contamine debe correr con los costos de dicha contaminación (el que contamina paga). De esta última responsabilidad se puede desprender una relación directa de medidas ambientales con el devenir del comercio, toda vez que "el responsable al costear el daño causado por sus actividades puede transferir ese costo a los consumidores en el precio de los correspondientes productos que ofrece, desalentando el consumo de esos bienes cuya protección genera precisamente gran contaminación" (PNUMA, 2001, p. 10).

Así mismo, la Declaratoria de Río consagra en el principio 12 que las medidas de política comercial con fines ambientales no deben constituir medios discriminatorios o que disfracen restricciones al comercio internacional. Y en tal sentido, insta a que los países establezcan, a través de consenso internacional, las medidas destinadas a tratar los problemas ambientales transfronterizos. Se evidencia así, la preocupación de muchos países respecto a la posibilidad de convertir el tema ambiental en obstáculo para sus relaciones comerciales.

La Agenda o Programa 21, por su parte, contempla cuestiones operativas que han de ejecutar, tanto a nivel internacional como nacional, todos los Estados Miembros después de finalizada la Cumbre de Río. La primera sección de este documento comprende las acciones enmarcadas en las dimensiones económicas y sociales, donde se señalan aspectos relacionados con la cooperación internacional para acelerar el desarrollo sostenible de los PED, la lucha contra la pobreza, la dinámica demográfica y la sostenibilidad, la integración del medio ambiente y el desarrollo en la adopción de decisiones, entre otros (Naciones Unidas, 1992).

La segunda sección trata sobre la conservación y gestión de los recursos para el desarroIlo, planteándose temáticas como la protección de la atmósfera, el cese de la deforestación, la conservación de la diversidad biológica y el fomento de la agricultura sostenible. La sección tercera habla del fortalecimiento de los "grupos principales", donde se destaca el capítulo que contempla el papel del comercio y la industria en la consecución de una "producción limpia". ${ }^{1}$ En la cuarta y última sección están las medidas de ejecución, allí aparece el tema de la transferencia de tecnología y de los mecanismos de cooperación internacional para aumentar la capacidad nacional en los PED, que en el ámbito comercial internacional cobran fundamental relevancia, como se comentará más adelante.

Otro de los logros fundamentales de la Cumbre de Río es la firma de la Convención Marco de las Naciones Unidas sobre el Cambio Climático (cMNUCc), cuyo principal objetivo, según el artículo 2, consiste en:

El texto del capítulo 30, sección III de la Agenda 21, dice que estos: "son esenciales para el desarrollo económico y pueden cumplir una función fundamental en la reducción de la utilización de recursos y el daño ambiental. Los Gobiernos, las empresas y las industrias (incluidas las empresas transnacionales) deberán fomentar una producción más eficaz y limpia y con una mayor reutilización y reciclaje de residuos y reducción de la cantidad de desechos que se vierten". 
La estabilización de las concentraciones de gas de efecto invernadero en la atmósfera a un nivel que impida interferencias antropógenas peligrosas en el sistema climático... ese nivel debería lograrse en un plazo suficiente para permitir que los ecosistemas se adapten naturalmente al cambio climático, asegurar que la producción de alimentos no se vea amenazada y permitir que el desarrollo económico prosiga de manera sostenible.

En los compromisos de la Convención se destaca el trato especial dirigido a los PED, cuyas economías sean vulnerables a las medidas ambientales que busquen mitigar el cambio climático, como por ejemplo:

Economías que dependan en gran medida de los ingresos generados por la producción, el procesamiento y la exportación de combustibles fósiles y productos asociados de energía intensiva, o de su consumo, o del uso de combustibles fósiles cuya sustitución les ocasione serias dificultades (art. 4 num. 10).

Y, de todos modos, bajo la cláusula de las responsabilidades comunes pero diferenciadas, la cMnucc establece que el esfuerzo de los PD debe ser mucho mayor que el de los PED.

\section{B. El Protocolo de Kioto y los mercados de carbono}

El principal instrumento negociado por la cMNUCC es el Protocolo de Kioto (1997), del que son Estados parte los grandes emisores de gases efecto invernadero (GEI), con excepción de Estados Unidos (a pesar de que es el segundo emisor más importante después de la China). En térmi- nos prácticos, el Protocolo de Kioto estableció unos objetivos de reducción de emisiones para 37 países industrializados (anexo I), los cuales se comprometieron a reducir dichas emisiones para el 2012 en un 5\% respecto de los niveles registrados en 1990, siendo esta una cifra de reducción global (Aguilar, Bouzas y Molinari, 2010). Sin embargo, de acuerdo con el nivel de responsabilidad de cada país, hay algunos cuyo porcentaje es superior a ese $5 \%$, como es el caso de la UE y Japón con un $8 \%$ y $6 \%$, respectivamente (Pérez, 2012). Para que los países pudieran cumplir con sus correspondientes metas de reducción, el Protocolo creó unos "mecanismos de flexibilidad" 2 para intercambiar los llamados créditos de carbono.

Los créditos de carbono son títulos que contienen "derechos de emisión" (derecho a contaminar), y que se pueden negociar en un mercado llamado, precisamente, "mercado de derechos de emisión" (art. 17, Protocolo de Kyoto). Estos créditos o bonos representan las unidades de contaminación a que tiene derecho cada país luego de restado el porcentaje de reducción a que se ha comprometido (Pérez, 2012). En el mercado de derechos de emisión se desarrollan los mecanismos económicos propuestos por Kioto, para que los países cumplan con sus metas individuales de reducción. En primera instancia está la negociación de bonos entre PD con compromisos de reducción, permitiendo a aquellos que superen sus compromisos vender

2 "para estimular las inversiones en países no industrializados o con economías en transición con el fin de facilitar la reducción de las emisiones de manera eficaz desde el punto de vista económico" (Portal de la Labor del Sistema de las Naciones Unidas sobre el Cambio Climático). 
los créditos excedentes a quienes no logren alcanzarlos (Aguilar et al., 2010). El otro mecanismo de negociación es el sistema de "implementación conjunta", consignado en el Protocolo de la siguiente forma:

Toda Parte incluida en el anexo I podrá transferir a cualquiera otra de esas Partes, o adquirir de ella, las unidades de reducción de emisiones resultantes de proyectos encaminados a reducir las emisiones antropógenas por las fuentes o de incrementar la absorción antropógena por los sumideros de los gases de efecto invernadero en cualquier sector de la economía (art. 6).

Afirma Aversano (2006) al respecto, que:

Se refiere a la posibilidad de que un país Anexo I financie proyectos de reducción de emisiones en otro país de Anexo I que se encuentre en transición hacia una economía de mercado, y tal reducción sea atribuida y contabilizada al primero (p. 9).

Este mecanismo beneficia a los dos países, pues el que financia aumenta su tenencia de "unidades de reducción de emisiones", a un costo inferior a si hubiese hecho la inversión en su territorio, y el segundo recibe inversión directa y se queda con la tecnología utilizada (Pérez, 2012).

Finalmente, en el artículo 12 del Protocolo de Kyoto se plantea el Mecanismo para el DesarroIlo Limpio (MDL), mediante el cual un PD puede invertir en proyectos de captura y reducción de emisiones ejecutados en un PED, recibiendo con ello certificados de reducción que puede acu- mular en su canasta de compromisos (Pérez, 2012). Los MDL incluyen a los países de América Latina y, según Aguilar et al. (2010), hoy es el principal mecanismo del mercado para captar recursos privados destinados a proyectos de mitigación. En el caso de Colombia, por ejemplo, hasta el año 2012 se habían registrado 12 proyectos MDL ante la oNu, que representan ingresos anuales de alrededor de 85 millones de dólares, ubicando este negocio en la veintidosava exportación más importante de este país (Biotun, 2008).

Frente a la llegada del 2012 y, por ende, al vencimiento del primer período de compromisos de Kioto, se planteó el acuerdo de un segundo período para los países del Anexo I, que empezó en el 2013 y finiquitará en el 2020. Así quedó estipulado en la Cumbre Climática del 2012 celebrada en Doha, donde Estados Unidos, Rusia, Canadá y Japón no firmaron está extensión (Semana, 2013). Sin embargo, solo se habló del fracaso en que, como se preveía, se convirtió el esperado Río + 20. Por ejemplo, Farooq Ullah, director ejecutivo de Stakeholder Forum señaló que: "hay una grave falta de especificidad en el documento aprobado sobre cómo exactamente proporcionaremos desarrollo sostenible, sobre cómo se va a financiar y sobre lo que realmente es la economía verde" (s.f.). Y, efectivamente, no se llegó a un acuerdo respecto a la economía verde y al marco institucional necesario para garantizar el desarrollo sostenible mundial. De acuerdo con la revista Semana de Colombia:

Las diferencias entre países de los hemisferios norte y sur, la presión de la crisis econó- 
mica en Europa, el temor y la falta de recursos para asumir los costos de una transición hacia economías menos destructivas del medio ambiente llevaron a los delegados a acordar un documento que niveló por lo bajo las tensiones entre los gobiernos, pero que deja sin respuestas concretas los principales desafíos que enfrenta un planeta cada vez más incapaz de sostener la carga humana que lo está consumiendo vorazmente (2012).

Por otra parte, se ha dado un trabajo paralelo al de los países del Anexo I, con todos los Estados Partes de la cmnucc (incluido Estados Unidos), donde se plantea una cooperación a largo plazo, proceso que se realizará en el marco de un órgano subsidiario de la Convención denominado Grupo de Trabajo Especial sobre la cooperación a largo plazo (AWG-LCA, por sus siglas en inglés). En su seno se desarrolló el Plan de Acción de Bali, que traza el camino a seguir en materia de mitigación, financiamiento y creación de capacidades (Naciones Unidas, 2008, p. 3).

\section{LOS AMUMA}

Son pocos los Amuma que reglamentan, o contienen disposiciones acerca del comercio internacional. El Manual de Medio Ambiente y Comercio (PNUMA, 2001) contiene una lista de los AmUMA más importantes respecto del planteamiento de disposiciones comerciales. El primero en mencionarse allí es la Convención sobre el Comercio Internacional de Especies Amenazadas de Fauna y Flora Silvestres (CITES), que se encarga de controlar el comercio de especies amenazadas a través de prohibiciones o licencias parciales. Enseguida cita al Protocolo de Montreal, que lle- va a cabo el control de aquellas sustancias químicas industriales que causan efectos nocivos en la capa de ozono de la estratosfera; así como al Convenio de Basilea, regulatorio del movimiento transfronterizo de desechos peligrosos y su eliminación, donde las partes han acordado, por ejemplo, la prohibición de exportación de desechos peligrosos provenientes de países miembros de la Organización para la Cooperación y el Desarrollo Económicos (OCDE) a países que no lo son.

También está el cDB, cuyos objetivos son la conservación de la diversidad biológica, el uso sustentable de sus elementos, así como la promoción de la participación justa y equitativa de los beneficios derivados del uso de los recursos genéticos (art. 1), y la cmnucc cuya principal batalla ha sido establecer estrategias para que las tecnologías de etapa final puedan reducir las emisiones de gases efecto invernadero. Y, finalmente, el Protocolo de Cartagena sobre Bioseguridad que, siendo adicional al CDB, trata sobre el intercambio comercial de los organismos vivos genéticamente modificados (oGMs), y los riesgos que estos pueden traer a la diversidad biológica; así mismo plantea un sistema de acuerdo previo fundamentado para los ogms que quieran ser introducidos al medio ambiente, y un procedimiento por el cual los países deciden si quieren restringir su importación. 


\section{EL MEDIO AMBIENTE EN EL COMERCIO}

La interacción entre comercio y medio ambiente tiene algunos intentos iniciales en el seno del Acuerdo General sobre Aranceles Aduaneros y Comercio (GATT, por sus siglas en inglés), donde se creó, por ejemplo, en 1971, el Grupo de las Medidas Ambientales y el Comercio Internacional, como un espacio de discusión con respecto a la posibilidad de que eventuales políticas ambientales de los países pudieran derivar en barreras proteccionistas para la liberalización del comercio mundial (Rojas, 2006). Sin embargo, este y otros intentos previos no fueron lo suficientemente significativos al respecto como sí lo fue lo hecho en la Ronda Uruguay (1986-1993), ${ }^{3}$ donde el tema ambiental se introduce definitivamente en el marco multilateral del comercio. Finalizada oficialmente la ronda con el Acuerdo de Marrakech, se crea la omc como organismo internacional que administra las normas relativas al comercio entre los países, y, a través de ella, se establecerá el Comité de Comercio y Medio Ambiente (сcMA), como se verá más adelante.

\section{A. La OMC y el CCMA}

Dentro del marco de la omc, en el mismo preámbulo del Acuerdo de Marrakech, el medio am-

3 En el GATT se manejaban una serie de rondas de negociaciones comerciales, donde, principalmente, se buscaba la reducción y eliminación arancelaria, aunque también se incluían negociaciones sobre las llamadas disciplinas antidumping, acuerdos sobre obstáculos técnicos al comercio, propiedad intelectual, entre otras. La Ronda de Uruguay puede considerarse como la última ronda del antiguo GATT, pues al crearse a partir de ella la omc como órgano rector del comercio mundial, este pasó a ser un instrumento más administrado por la omc. biente cobra especial importancia cuando se habla de "la utilización óptima de los recursos mundiales de conformidad con el objetivo de un desarrollo sostenible y procurando proteger y preservar el medio ambiente e incrementar los medios para hacerlo". De igual forma, en la Decisión sobre Comercio y Medio Ambiente, las partes encomendaron al Consejo General de la omc la creación del сcmA, al cual se le prescribió la labor de determinar la relación existente entre las medidas comerciales y aquellas de carácter ambiental, con el fin de promover el desarrollo sustentable; así como la elaboración de recomendaciones sobre posibles modificaciones a las disposiciones del sistema multilateral del comercio relacionadas con el ambiente.

Las funciones del CCMA, en principio, se limitan a establecer estudios de asuntos ambientales relacionados con las políticas de la omc; en otras palabras, aquellas quedan restringidas a la determinación de los posibles efectos de algunas medidas ambientales en el comercio, o, por lo menos, sobre las reglas mínimas que en materia comercial consagra el sistema multilateral (Galeano, 2006). Si bien la Decisión establece que no debe haber contradicción entre el ámbito jurídico ambiental y el comercial, como dice Zelada (2003), eso no significa que en la realidad no se presente dicha contradicción; por lo cual, se considera importante establecer el nivel de compatibilidad y de supremacía entre una norma ambiental y una comercial multilateral basada en la liberalización y no discriminación del comercio.

Por otro lado, dentro de la omc hay tres anexos que son claves para la interacción comercio/ 
medio ambiente, a saber: el GaTT, el Acuerdo sobre Obstáculos Técnicos al Comercio y el Acuerdo sobre la Aplicación de Medidas Sanitarias y Fitosanitarias.

\section{El GATT}

El gatT contiene las cláusulas de la "nación más favorecida" 4 y del "trato nacional" 5 que materializan el principio de la no discriminación, fundamental para el marco multilateral en los procesos de liberalización del comercio en materia de aranceles aduaneros. Se ha puesto especial atención al artículo 3, pues contiene la cláusula del trato nacional, por la cual los productos importados deben recibir el mismo trato de sus similares fabricados a nivel local, donde la acepción "producto similar" puede tener ciertas implicaciones en el ámbito ambiental. Según el PNUMA (2001), pueden presentarse en frontera, por ejemplo, dos productos iguales o "similares", no obstante, uno ha sido fabricado en un proceso productivo altamente contaminante y el otro no, así como pueden ser "similares" a un producto nacional no contaminante; de considerar como "similares" a todos los productos, no podría discriminarse a aquel contaminante en aras de preferir al que no contamina, pues se

4 Contenida en el artículo 1. A través de ella un país en el ejercicio de negociación bilateral con otro país, una vez establezca una ventaja arancelaria o de otra índole sobre un producto del segundo país, automáticamente le otorga dicha ventaja al resto de los países partes del tratado, haciéndose efectivo con ello, además, el carácter sustancialmente multilateral del acuerdo.

5 Siendo complementaria de la cláusula de la nación más favorecida, exige que una vez un producto ha traspasado la frontera y se encuentra en el mercado interno de otro país, debe recibir el mismo trato de los productos nacionales, en términos de precios, contratos, impuestos y demás. estarían violando los principios de la no discriminación y del trato nacional.

Para determinar la similitud entre dos productos, el "sistema de solución de diferencias" de la omc ha desarrollado algunos criterios, a saber: el uso final en un mercado determinado; los gustos y hábitos del consumidor; las propiedades, naturaleza y calidades de los productos; y, finalmente, la posibilidad de sustitución comercial de un producto por el otro (PNUMA, 2001, p. 31).

El artículo 20 del GaTT también resulta relevante en materia ambiental. En él se contemplan las excepciones al cumplimiento de las normas comerciales multilaterales. Dos literales de la disposición son atinentes al tema del medio ambiente: el b), sobre la protección de la salud y la vida de las personas y de los animales, así como la preservación de los vegetales; y el g), acerca de la conservación de los recursos naturales agotables. Sin embargo, para que los países puedan aplicar estas excepciones deben probar que, efectivamente, se cumplen las condiciones para hacerlo, y bajo una "justificación provisional" inicial y una "justificación final" definitiva. La "justificación provisional" para acudir inicialmente a la aplicación de los literales b) y g), y la "justificación final” para probar que con la excepción a aplicar no se está violando el párrafo introductorio del artículo $20,{ }^{6}$ es decir, que tales excepciones no constituyen obstáculos

6 El párrafo introductorio del artículo XX reza: "A reserva de que no se apliquen las medidas enumeradas a continuación en forma que constituya un medio de discriminación arbitrario o injustificable entre los países en que prevalezcan las mismas condiciones, o una restricción encubierta al comercio internacional, ninguna disposición del presente Acuerdo será interpretada en el sentido de impedir que toda parte contratante adopte o aplique las medidas". 
encubiertos al libre comercio ni violaciones al principio de no discriminación.

Para la aplicación del literal g), por ejemplo, el Estado parte debe probar que la legislación propia regula la conservación de los recursos naturales agotables, estableciéndose restricciones locales a la administración, producción o consumo del recurso a conservarse, así como el fin pleno de la conservación (PNUMA, 2001).

\section{El Acuerdo sobre Obstáculos Técnicos al Comercio}

Sobre el Acuerdo sobre Obstáculos Técnicos al Comercio (отс) hay que decir que son reglamentos de carácter no comercial pero que, potencialmente, pueden afectarlo. Los llamados "obstáculos" están relacionados con medidas que pueden considerarse necesarias por los países para asegurar la calidad de sus exportaciones, para la protección de la salud y la vida de las personas y los animales, o para la preservación de los vegetales en la protección general del medio ambiente (Galeano, 2006). Hay reglamentos de contenido ambiental como, por ejemplo, aqueIlos que exigen que los productos forestales provengan de bosques tratados de manera sustentable. El mismo Acuerdo oтc establece qué tipo de obstáculos técnicos son permitidos vía estos reglamentos, así como los requisitos que deben cumplir para serlo, para "evitar que a través de reglamentos técnicos sean creados obstáculos innecesarios al comercio internacional" (numeral 2.2 art. 2).

\section{El Acuerdo sobre la Aplicación de Medidas Sanitarias y Fitosanitarias}

Por su parte, el Acuerdo sobre la Aplicación de Medidas Sanitarias y Fitosanitarias (AMSF) contiene "las normas básicas para garantizar la protección de los seres humanos, animales y vegetales respecto del peligro que pueda acarrear el traslado de animales, plantas y productos alimenticios en el marco del comercio internacional" (PNUMA, 2001, p. 34). El Acuerdo señala cuándo se debe permitir la aplicación de estas medidas así como los requisitos que deben cumplirse y la sustentación científica que ha de respaldarlas.

\section{B. Conferencias ministeriales y la Declaración de Doha}

Con posterioridad a la entrada en vigencia de la omc, se han celebrado conferencias ministeriales que han tocado con distintos niveles de intensidad el tema ambiental; los resultados, para muchos, son bastante precarios respecto a los alcances que deberían lograrse en el marco de estas reuniones del sistema multilateral de comercio.

En la Conferencia Ministerial llevada a cabo en Singapur en el año de 1996, el Informe del сcмA refleja la preocupación de algunos PD frente a las medidas ambientales que puedan afectar la competitividad y las oportunidades de acceso a los mercados de las Pymes, señalando, por ende, la importancia de cooperar con lOS PED para que comiencen a implementar políticas de desarrollo conectadas con el medio ambiente 
(Galeano, 2006). Con ello se evidencia la necesidad de lograr una sintonía entre la defensa del medio ambiente, las políticas de desarrollo y crecimiento económico y el libre comercio.

Así mismo, en la Cuarta Conferencia Ministerial de Doha, en el 2001, países en vía de desarrollo como la India pidieron a la ccma centrarse especialmente "en el efecto de las medidas medioambientales, en el acceso a los mercados, en las disposiciones pertinentes del Acuerdo sobre los $\mathrm{ADPIC}^{7}$ y en las prescripciones relativas al etiquetado para fines medioambientales" (Galeano, 2006, p. 291). Los párrafos 31, 32 y 33 de la Declaración Ministerial se ocupan del tema de comercio y medio ambiente, cuyos subtemas principales son: los Amuma y su interacción con las normas de la omc, así como los obstáculos al comercio de bienes y servicios ecológicos (omc, 2001).

De acuerdo con Kumar y Chowdhury (2005), si hay algún conflicto por medidas ambientales entre algún Amuma y normas de la omc, este debe resolverse en el marco del órgano de solución de diferencias y guiándose por la jurisprudencia de la omc existente para casos ambientales, lo cual conduce a decir que solo sería admisible que una norma ambiental infrinja alguna de la омc, si pasa la prueba como una de las excepciones ambientales previstas en el artículo XX del GATT. Por tal razón, partes como la UE, Japón, Suiza y Noruega presentaron en Doha propuestas para modificar dicho artículo (XX) y acercar

7 Acuerdo sobre los Aspectos de los Derechos de Propiedad Intelectual relacionados con el Comercio. más los acuerdos de la omc y los Amuma. Estos países realizaron una exposición acerca de sus actividades con los AmumA, señalaron experiencias en su negociación, así como la aplicación de las obligaciones comerciales que adquirieron con ellos.

La India, así mismo, resaltó la importancia, como mecanismo de cooperación entre países, de intercambiar información sobre las experiencias nacionales o locales en políticas ambientales, siendo la existencia y la aplicación del Protocolo de Montreal el ejemplo de éxito a mostrar de esas medidas de apoyo y cooperación (Kumar y Chowdhury, 2005). Cabe aclarar que el exponer dichas experiencias nacionales buscaba demostrar cómo se pueden aplicar medidas ambientales sin afectar negativamente al comercio.

Otra información de interés que analizan Kumar y Chowdhury (2005) en el marco de las negociaciones de Doha, tiene que ver con las "listas de productos o bienes ambientales", compilada por la Secretaría de la omc a petición de la cсмA y que, en principio, han sido elaboradas por instituciones como la OCDE y el Foro de Cooperación Económica Asia-Pacífico (APEC, por sus siglas en inglés). En ellas se revelan datos interesantes del comercio de bienes ambientales entre los PED y los PD. Por ejemplo, se encuentra que los PED Son netos importadores de bienes ambientales de la lista APEC, mientras que solo dos países son exportadores netos de bienes ambientales en la lista OCDE; los PD presentan más del $80 \%$ de las exportaciones mundiales, mientras que la participación de los PED es del 15\%; y los PED, 
como grupo, son exportadores netos de 26 de los 182 bienes ambientales en el conjunto de la OCDE y las listas del APEC.

El documento de estos autores señala, además, que los PED son significativos exportadores de gas natural y de productos ambientalmente preferibles, como alfombras de lana, hilados y tejidos, caucho natural, vegetales, madera y sus derivados, entre otros. Cuestión relevante si se tiene en cuenta la creciente tendencia a preferir la importación y consumo de productos amigables con el ambiente.

Sin embargo, en las negociaciones en listas de bienes ambientales, con el objetivo de que sean incluidos en una eventual liberalización comercial sobre la base de la "nación más favorecida", no se ha llegado a acuerdos que dejen satisfechos a todos los países. Para Rojas (2006), por ejemplo, efectivamente no hay consenso en la definición de "bienes ambientales", y el sistema de listas si bien ostenta un carácter de simplicidad, no ha logrado dicho consenso, pues ha demostrado ser insuficiente ... debido en especial a su incapacidad para cumplir plenamente con los objetivos establecidos en el mandato de Doha: la liberalización comercial, la protección del medioambiente y la promoción del desarrollo sobre la base de una mayor participación de los países en desarrollo y menos adelantados y a partir de un trato especial y diferenciado (p. 22).

En general, es posible concluir que las negociaciones sobre comercio y medio ambiente en la oMc no han tenido éxito en cuanto a consensos. Sin embargo, en este panorama de ausencia de consensos, se destaca la participación activa y propositiva de países emergentes como la India, Brasil y Sudáfrica, al menos para apoyar o reservarse frente a las reuniones del ccmA.

\section{EL MEDIO AMBIENTE EN EL COMERCIO REGIONAL Y BILATERAL}

En este punto del análisis se revisarán algunos apartes de las experiencias regionales de la relación comercio/medio ambiente. Así mismo, se citarán "casos locales" sobre cómo se ha afrontado esta interacción en niveles nacionales, vale decir, países que se enfrentan individualmente al tema medioambiental, a propósito de negociaciones bilaterales de libre comercio.

\section{A. La experiencia de la Unión Europea}

De acuerdo con Galeano (2006), en la UE y los Estados Unidos se considera, a juzgar por lo que expusieron en la Tercera Conferencia Ministerial de la omc en Seattle (1999), que las normas comerciales y ambientales deben apoyarse mutuamente para favorecer el desarrollo sostenible. Antes de iniciarse determinadas negociaciones comerciales, los países no solo hacen una evaluación del impacto económico de estas sino que, además, deben evaluar cómo afectan también las estructuras sociales y del medio ambiente. Por supuesto, el alcance y el enfoque de dichas evaluaciones debe darse por el respectivo país, de acuerdo con sus prioridades en materia de desarrollo, políticas socioeconómicas y del medio ambiente (PNUMA, 2001). Dichas evaluaciones deben mantenerse en el transcurso 
de las negociaciones comerciales, no solo como forma de encontrar los problemas potenciales, sino, además, de indagar las posibles medidas para mitigarlos; así como para lograr, de ser posible, la introducción de salvaguardas y excepciones en dichas negociaciones.

Sabemos que la UE es la organización internacional más desarrollada, y el mejor ejemplo de integración regional del que pueda hacerse mención, siendo una unión aduanera y mercado común a la vez. Los aspectos medioambientales están contenidos en las llamadas "directivas" de la UE, que permiten trazar los objetivos a alcanzar pero otorgando la libertad a los Estados de escoger los medios para cumplirlos (PNUMA, 2001, p. 86). Es así como las directivas regulan temas sobre: normas de emisión, gestión de desechos peligrosos y domiciliares, envase y embalaje, emisiones atmosféricas provenientes de plantas y vehículos, normas de la calidad del aire y la capa de ozono, protección de la naturaleza, contaminación acústica, especies amenazadas, cambio climático, entre muchos otros.

Respecto al cambio climático cuenta, por ejemplo, con la Decisión 406/2009/CE del Parlamento Europeo y del Consejo (Decisión 406/2009/CE), que fue suscrita por los Estados miembros de la ue para reducir sus emisiones de GEl, con el objetivo de poder cumplir los compromisos adquiridos en la materia por parte de la Comunidad para el 2020 (Paquete 2020 de la UE). Dicha Decisión establece una meta de reducción global de las emisiones del 20\% para el 2020 sobre el nivel de 1990 -recordemos que la meta del Protocolo de Kioto para el 2012 era del 5\%, también sobre el nivel de 1990-. Así mismo, espera que para el mismo año se haya incrementado en un $20 \%$ la participación de energías renovables en la matriz energética (Diario Oficial de la Unión Europea, 2009).

Las medidas más importantes que estipula la UE al respecto, son: la revisión del esquema europeo de emisiones, una directiva para promover la energía de fuentes renovables, normas de calidad ambiental para combustibles y biocombustibles, una extensión de los objetivos nacionales vinculantes de las emisiones a sectores no regulados por el actual régimen de comercio de derechos de emisión (transporte, agricultura, construcción, entre otros), y la revisión de una directiva sobre estándares para automóviles (Aguilar et al., 2010).

Con esta nueva norma, a partir del 2013, el mercado europeo de créditos de carbono cubre todas las fuentes intensivas en el uso de energía, y los países de la comunidad adoptan medidas para ampliar la cobertura del mercado de emisiones. Se prevé que el mecanismo para asignar las emisiones será el remate de emisiones. El Banco Mundial (citado por Aguilar et al., 2010) estima que el remate de emisiones tiene el potencial de generar 25-40 mil millones de euros anuales, cuyo 50\% será usado para promover un crecimiento resistente al clima y bajo en carbono, mientras que el otro 50\%, sin tener un fin asignado, podría destinarse para compensar el costo de cumplir con determinada legislación por parte de sectores sensibles, donde los países de Latinoamérica con mayor potencial de mitigación podrían tener participación. 
Una oportunidad para Latinoamérica, respecto de estas políticas ambientales de la Comunidad Europea, está relacionada con el aumento en un $10 \%$ del uso de biocombustibles en el transporte, que debe ser cubierto con importaciones de productos como el biodiesel y el bioetanol, provenientes, seguramente, de países de la región. Sin embargo, hay un reto de por medio: la ue exige, a través de normas de calidad, que para ser considerados renovables, los biocombustibles deben cumplir con criterios sobre protección de la biodiversidad, las especies y los ecosistemas, así como respecto a una cantidad tope de emisiones de GEI (Aguilar et al., 2010).

\section{B. El CaSO TLCAN}

El Tratado de Libre Comercio de América del Norte (TLCAN), celebrado entre Canadá, México y Estados Unidos, fue firmado en el año 1994. En su seno se aprobó el Acuerdo sobre Medio Ambiente, creador, a su vez, de la Comisión para la Cooperación Ambiental (CCA). La ccA puede resolver diferencias entre los tres países, especialmente cuando alguno, bajo un beneficio comercial, incumple reiteradamente una normativa ambiental (PNUMA, 2001). El TLCAN enumera cinco acuerdos ambientales internacionales, cuyas obligaciones, según dice, deben prevalecer sobre el tratado en caso de incompatibilidad. Entre dichos acuerdos están el Protocolo de Montreal, el Convenio de Basilea y la CITES (art. 104 TLCAN).

Sin embargo, Galeano (2006) encuentra datos contradictorios sobre el tema ambiental entre Ios Estados miembros del tLCAN. Así, por ejemplo, la contaminación atmosférica muestra incre- mentos en los niveles de monóxido de carbono y bióxido de azufre (SO2) en Estados Unidos y de SO2 en México, así como reducciones considerables de la contaminación atmosférica en los sectores papeleros de Canadá y México; y los desechos peligrosos muestran un incremento considerable en lugares de Canadá y una reducción en algunos estados del norte de Estados Unidos.

También, a pesar de la existencia de la cca y de la autonomía estatal otorgada para la reglamentación ambiental, el TLCAN no permite el logro de un mayor comercio de energía limpia, toda vez que:

Alienta los subsidios al desarrollo de los combustibles fósiles, impide que los gobiernos nacionales regulen la tasa de explotación de los recursos y estimula procesos de desregulación, donde se da prioridad a las empresas transnacionales por encima de la conservación y protección del medio ambiente (Galeano, 2006, p. 297).

Dentro de los países del TLCAN, Estados Unidos es el que parece tener mayor interés en implementar medidas ambientales a nivel local, a pesar de la falta de compromiso en el sistema multilateral. Son conocidos los subsidios a la inversión en energías limpias, y se destaca el proyecto de Ley Waxma-Markey sobre Energía Limpia y Seguridad en América (American Clean Energy and Security Act -ACES). De acuerdo con la ACES (citada por Aguilar et al., 2010), esta busca la creación de puestos de trabajo en la producción de energías más limpias y menos costosas, así como incentivar la independencia energética 
y reducir el calentamiento global. Este proyecto de ley establece, también, dos mercados de emisiones: uno para la mayoría de Geı y otro para los hidrocarburos; ambos fijando un límite a las emisiones anuales para que las entidades reguladas obtengan permisos de emisión hasta esos límites.

El proyecto de ley ACES propone una reducción de emisiones de Geı para toda la economía del 20\% en el 2020, $42 \%$ en el 2030 y $83 \%$ en el 2050 (Natural Resources Defense Council, 2009, p. 2). Según Aguilar et al. (2010)

La meta global se lograría mediante el otorgamiento de un número limitado de permisos que se distribuirían a través de un sistema de remates entre las entidades cubiertas como plantas generadoras de energía eléctrica, productores e importadores de combustibles y gas natural, fuentes industriales fijas, instalaciones industriales movidas por combustibles fósiles, etc. (p. 16).

Por último, es de resaltar en el proyecto que para tener los mencionados créditos por actividades que reduzcan o eviten emisiones GEI en PED, debe existir de por medio un acuerdo bilateral o multilateral con los Estados Unidos.

Recientemente el presidente estadounidense, Barack Obama, a través de la Agencia de Protección Medioambiental, anunció una política pública al respecto, cuyo objetivo es la reducción de emisiones de carbono en un 30\% para el año 2030, basándose primordialmente en la disminución de la producción de energía a base del carbón (Semana sostenible, s.f.). Busca propi- ciar, además, la innovación tecnológica para la producción de energía de fuentes renovables e intenta incluir nuevamente el sistema de créditos de carbono, quizás en los términos del mismo proyecto ACES. No obstante, la iniciativa es, por ahora, un documento abierto a la discusión y a su perfeccionamiento, pero con la intención de implementarse a partir del año 2016.

\section{América Latina frente a las iniciativas ambientales de la Unión Europea y los Estados Unidos}

Respecto al crecimiento de las políticas y medidas ambientales tanto en la UE como en los Estados Unidos, América Latina, eventualmente, podría verse afectada en las exportaciones hacia cada uno de estos destinos, toda vez que, como hemos observado, hay proyectos ambientales (no comerciales) que pueden restringir y obstaculizar el comercio. Dentro de las medidas a temer están los mecanismos de ajuste en frontera, la aplicación de reglamentos y estándares técnicos, y los etiquetados voluntarios.

Los mecanismos de ajuste en frontera buscan evitar la fuga de carbono y la pérdida de competitividad, imponiendo a los productos importados costos similares a los que tienen los productos internos para el cumplimiento del régimen de comercio de emisiones, utilizando, por ejemplo, la imposición de "precios justos" (PNUMA y омc, 2009). Por su parte, los reglamentos y estándares técnicos buscan mejorar los procesos productivos en términos ambientales, como es el caso de las normas de calidad sobre combustibles y biocombustibles. Y los etiquetados vo- 
luntarios constituyen un mecanismo que provee información a los consumidores sobre la naturaleza de los productos en cuanto a su contenido de carbono (footprint), la distancia recorrida entre el lugar de producción y el de consumo (foodmiles), así como la medición de la "huella de carbono" o producción de gases de efecto invernadero generada durante el ciclo de vida del producto, lo cual en el sector alimenticio puede convertirse en una verdadera barrera de acceso a los mercados (Aguilar et al., 2010).

Las industrias más contaminantes, desde el punto de vista climático, son las correspondientes al hierro, el acero, el aluminio, el cemento, el papel y los químicos, dentro de las cuales los productos más sensibles en el comercio entre América Latina y la ue son el hierro y el acero, cuyas exportaciones alcanzaron un promedio anual de casi 14 mil millones de dólares entre 2006 y 2008 (Aguilar et al., 2010). Comparando el nivel contaminante de la producción de acero en distintas latitudes, se encuentra que la de Estados Unidos es la menos intensiva en carbono del mundo, mientras que las de México y Brasil son altamente intensivas, superando con creces, a su vez, las emisiones de la ue (Houser, Bradley, Childs, Werksman y Heilmayr, 2008).

Al parecer, los países de la región que podrían verse más afectados por estas medidas ambientales, aplicadas en sus flujos comerciales, serían México y Brasil. Ambos por el amplio valor de comercio comprometido, México con los Estados Unidos y Brasil con la UE. El mayor impacto podría producirse, entonces, en la industria del acero. Sin embargo, se precisan mayores estu- dios minuciosos de las emisiones asociadas a estas actividades en cada país, y compararlas con las producidas en Estados Unidos y la UE, para determinar las condiciones de competitividad con que se cuenta en el tema. Dicen Aguilar et al. (2010) que en el caso del aluminio, el cemento y la producción del papel, las emisiones de los exportadores latinoamericanos parecen compararse favorablemente con las de los productores domésticos de los PD.

\section{El caso Mercosur}

El Mercosur es un acuerdo de integración subregional entre Brasil, Argentina, Uruguay, Paraguay y, recientemente, Venezuela. Funciona actualmente como una unión aduanera, es decir, todos los miembros tienen los mismos aranceles aduaneros aplicables al resto del mundo. Distintas decisiones del Consejo de Mercado Común se refieren a cuestiones relacionadas con el ambiente, tales como las políticas de energía, los plaguicidas, el transporte de productos peligrosos, entre otros. Se resalta, así mismo, la Reunión Especializada en Medio Ambiente, formada en el marco de la Declaración de Canela de 1992, para estudiar la legislación, normas y prácticas ambientales de los cuatro países miembros de ese entonces (Venezuela ingresó en el 2013). De la Reunión Especializada en Medio Ambiente surge el subgrupo $n .^{\circ} 6$ sobre Medio Ambiente, que ha tocado temas como la competencia, los obstáculos arancelarios al comercio y un sistema de información común sobre medio ambiente (PNUMA, 2001). 
En el 2001, con la Decisión 02/01 se aprobó el Acuerdo Marco sobre Medio Ambiente del Mercosur, cuyo objeto es "el desarrollo sustentable y la protección del medio ambiente, mediante la articulación de las dimensiones económicas, sociales y ambientales, contribuyendo a una mejor calidad del ambiente y de la vida de la población" (art. 4). En materia de cooperación ambiental el Acuerdo obliga a los Estados miembros a cumplir con las obligaciones de los acuerdos internacionales firmados en materia ambiental. También se destacan en el Mercosur los acuerdos de entendimiento sobre el ambiente, negociados con la ue y Canadá.

\section{E. El caso de un acuerdo bilateral en Latinoamérica}

Con miras a revisar un ejemplo local, o nacional, sobre la relación comercio y medio ambiente, se ha tomado el ejemplo de Chile, uno de los primeros países de la región en iniciar negociaciones de apertura económica con PD y cuya economía, hoy en día, es considerada una de las más sólidas del continente, incluyendo, además, interesantes intentos de desarrollo sostenible.

El modelo de exportación chilena se ha basado en la minería, la agricultura, la silvicultura y la pesca; por ello, desde temprano, ha habido preocupación por la sobreexplotación de estos recursos. Así mismo, las empresas chilenas, públicas y privadas, vienen invirtiendo en procesos productivos sustentables y tecnologías limpias desde la apertura económica de los noventa, mostrando una inclinación hacía políticas ambientales anticipadas, en vista de las nego- ciaciones de libre comercio que retaban a los empresarios chilenos con "requisitos verdes" provenientes de los mercados desarrollados (Gómez-Lobo, 1992).

La apertura chilena al comercio exterior en el sector industrial, según Gómez- Lobo (1992), tuvo un impacto positivo sobre el medio ambiente, porque industrias contaminantes se expandieron a tasas inferiores respecto de aquellas que, incluso, contaminan menos, lo cual puede explicarse por la incorporación de tecnologías limpias por parte de empresas transnacionales chilenas, que bajo presiones competitivas crecientes, fruto de la apertura, tuvieron que invertir y actualizarse en estas nuevas tecnologías.

El caso chileno sugiere, entonces, que el libre comercio puede tener impactos ambientales variados (no solo negativos). El Gobierno chileno, a partir de 1990, le ha apostado a la liberalización económica con crecimiento sustentable, donde los efectos nocivos tienden a ser menores gracias a la adopción de una visión estratégica de crecimiento económico y protección ambiental, evidenciándose así que, independientemente de la liberalización económica, es el modelo de desarrollo de un país el que determina el impacto al medio ambiente (Galeano, 2006).

\section{CONCLUSIONES}

En primer lugar hay que mencionar que la relación comercio/medio ambiente es distinta según el plano desde donde sea revisada, vale decir, las conclusiones que puedan extraerse de 
su análisis en el sistema multilateral son diferentes a aquellas deducibles de las experiencias regionales y aun locales.

En el sistema multilateral, enmarcado en el mero derecho ambiental internacional, encontramos el trabajo técnico más riguroso respecto a posibles implementaciones reales de medidas que protejan el medio ambiente, intentando, en últimas, devolverle al ecosistema mundial una dinámica sostenible. El sistema multilateral, en vista de que no podemos prescindir de la economía montada en los últimos cincuenta años, parte de la premisa del "desarrollo sostenible" en la que interesa un crecimiento económico de los países, comprometido con el derecho de las generaciones futuras de contar con los mismos recursos que tenemos nosotros en el presente.

En el marco de Naciones Unidas se destaca el trabajo de la cMnucc, con su proyecto de cambio climático más ambicioso: el Protocolo de Kioto. Con la desastrosa experiencia de Río +20 , Kioto nos muestra cómo propuestas viables en la teoría, no son ejecutadas o cumplidas en la realidad de la economía y el comercio de los países, siendo la falta de voluntad política y la presión de los grandes actores económicos las responsables de lo que podríamos considerar uno de los mayores fracasos del sistema multilateral, esta vez en su compromiso con el cambio climático, que, en últimas, es la obligación de recuperar, proteger y mejorar el medio ambiente y el planeta. En el marco multilateral también se destacan los AMUMA, como proyectos reguladores de campos específicos que, en escenarios como el comercio internacional, afec- tan o pueden afectar potencialmente el medio ambiente.

El tema, en el seno de la omc ha sido controvertido, toda vez que se acusa la falta de compromiso de esta organización para con las medidas ambientales, bajo el evidente temor de que estas se conviertan en serios obstáculos y restricciones al libre comercio entre los países.

En cuanto a las conclusiones extraíbles del análisis regional y local, podemos decir que la UE parece ser la más comprometida con medidas ambientales, destacándose el Paquete de Cambio Climático y Energía para el 2020, en el que la comunidad ha definido su proyecto individual de mitigación luego del 2012, año en que se venció el primer período de compromisos de reducción de Gel de los países del Anexo I del Protocolo de Kioto. Queda esperar los resultados de la implementación real y efectiva de los proyectos de medidas ambientales planteados, teniendo en cuenta el marco de la grave y ya larga crisis económica que atraviesa la comunidad.

En el caso del TLCAN parece que el tema ambiental tampoco ha tenido destacados alcances; no obstante, Estados Unidos, su principal miembro, destaca interesantes proyectos de medidas ambientales locales para aplicar en todos los sectores que contaminan el medio ambiente, lo cual puede resultar paradójico si lo contrastamos con la ausencia de compromiso en el sistema multilateral de quien es el segundo mayor emisor de Gel del mundo. 
En general, llama la atención que, bien se esté en el marco multilateral, regional o local, existe la intuición generalizada de que mayores y serios compromisos con las medidas ambientales en las industrias y en el comercio pueden afectar indefectiblemente la competitividad y el crecimiento de las economías de los países y sus empresas. Sin embargo, en este análisis se han expuesto estudios que al respecto resultan meramente especulativos, pues no es tan fácil prever el grado de afectación de las medidas ambientales sobre una economía, dado que, incluso, algunos casos demuestran la relación armónica que pueden llegar a tener el comercio y el medio ambiente.

Por ejemplo, en los datos encontrados sobre Chile, hay hipótesis que permiten plantear cómo la toma de medidas ambientales en la economía puede ser una herramienta constructora de competitividad y crecimiento económico en un país, con el añadido fundamental de estar practicando desarrollo sostenible y contribuyendo a mejorar el medio ambiente. Galeano (2006) afirma que:

Países en vía de desarrollo están aprendiendo que la política ambiental no es una fuente de problemas sino una fuerza impulsora de carácter innovador que fomenta el crecimiento económico y la competitividad, al fin y al cabo, ésta es la verdadera esencia del desarrollo sustentable (p. 299).

Otro punto a sintetizar se relaciona con la multiplicidad de organizaciones y acuerdos internacionales sobre la materia ambiental, que han hecho se difumine cualquier jerarquización nor- mativa, correspondiendo, de alguna manera, a la diversidad de intereses e incertidumbres de cada país. No obstante, lejos de mantener una incertidumbre frente a cuáles herramientas internacionales tomar, la situación puede permitir un grado de flexibilidad y adaptabilidad para los países con voluntad de contribuir favorablemente al medio ambiente, donde las experiencias locales o regionales pueden tener un papel más interesante, lejos de las rigurosidades de algunos mecanismos multilaterales (Keohane y Victor, 2010).

La interacción entre comercio y medio ambiente, de todas formas, es compleja y se evidencian tanto amenazas como oportunidades para los países, los mercados locales y las empresas. Se supone que el desafío para todos es aprovechar las oportunidades y reducir las amenazas. En este punto es donde los países latinoamericanos deben apostar a aprovechar las oportunidades de esta interacción, sobretodo porque, dadas sus condiciones geofísicas y su inmensa riqueza en recursos naturales renovables y no renovables (agua, bosques, páramos, biodiversidad, tierras fértiles y aptas para cultivos agrícolas de todos los tipos, metales, petróleo, gas natural, entre otros), tienen elementos de peso para negociar lo más conveniente no solo para su propio desarrollo económico, sino para algo más importante: el futuro del medio ambiente que es, en últimas, el futuro del planeta y de la humanidad. 


\section{Referencias}

Aguilar, S., Bouzas, R. y Molinari, A. (2010). Cambio climático y comercio internacional: algunas implicaciones para América Latina. Obtenido de http://www.udesa.edu.ar/ files/UAHumanidades/EVENTOS/Paperbouzas100510.pdf

Aversano, N. (2006). El calentamiento global: bonos de carbono, una alternativa. Obtenido de http://www.ingenieriaquimica.org/system/files/bonos_carbono.pdf

Diario Oficial de la Unión Europea. (5 de junio de 2009). Decisión n. ${ }^{\circ}$ 406/2009/CE del Parlamento Europeo y del Consejo de 23 de abril de 2009. Recuperado el 25 de febrero de 2014 de eir-lex.europa.eu: http://eur-lex. europa.eu/LexUriServ/LexUriServ.do?uri=0J :L:2009:140:0136:0148:ES:PDF

Gómez-Lobo, A. (1992). Las consecuencias ambientales de la apertura comercial en Chile. Colección Estudiantes cIEPLAN, (35), 107.

Houser, T., Bradley, R., Childs, B., Werksman, J. y Heilmayr, R. (2008). Leveling the Carbon Playing Field. International Competition and US Climate Policy Design. Washington: Peterson Institute for International Economics and World Resources Institute.

Keohane, R. y Victor, D. (2010). The Regime Complex for Climate Change. Obtenido de: http://ilar.ucsd.edu/assets/001/501988. pdf
Kumar, S. y Chowdhury, N. (2005). Trade and Environment in the wTo: Negotiating Options for Developing Countries. Obtenido de: http://www.ris.org.in/images/RIS_images/ pdf/dp103_pap.pdf

Galeano, J. (2006). Medio ambiente en el tratado de libre comercio. Prolegómenos-Derechos y Valores, IX (18), 285-304.

Naciones Unidas. (1987). Our Common Future. Obtenido de: http://conspect.nl/pdf/Our_ Common_Future-Brundtland_Report_1987. pdf

Naciones Unidas. (1992). Programa 21. Obtenido de: http://sustainabledevelopment. un.org/content/documents/1718a21_summary_spanish.pdf

Naciones Unidas. (1992). Convenio sobre la diversidad biológica. Obtenido de cbd: https:// www.cbd.int/doc/legal/cbd-es.pdf

Naciones Unidas. (14 de marzo de 2008). Convención marco sobre el cambio climático. Obtenido de Naciones Unidas: http:// unfccc.int/resource/docs/2007/cop13/ spa/06a01s.pdf

Natural Resources Defense Council -NRDC. (Mayo, 2009). El Acta de Energía Limpia y Seguridad (ACES) puede ayudar a impulsar la energía limpia y solucionar la crisis climática. Recuperado el 25 de febrero de 2014 de nrdc: ww.nrdc.org/laondaverde/globalWarming/legislation/files/waxman.pdf 
Pérez, A. (2012). Análisis de eficiencia del mecanismo de regulación propuesto por Kioto. [Tesis de grado, Universidad Industrial de Santander, Bucaramanga, Colombia].

Portal de la Labor de las Naciones Unidas sobre el Cambio Climático. Mecanismos que ayudan a reducir las emisiones. Obtenido de: http://www.un.org/es/climatechange/mechanisms.shtml

Programa de las Naciones Unidas para el Medio Ambiente -PNumA. (2001). Manual de medio ambiente y comercio. Obtenido de http:// www.iisd.org/pdf/envirotrade_handbook_ es.pdf

Programa de las Naciones Unidas para el Medio Ambiente y Organización Mundial del Comercio. (2009). El comercio y el cambio climático. Recuperado el 25 de febrero de 2014 de wTo: https://www.wto.org/spanish/res_s/ booksp_s/trade_climate_change_s.pdf

Organización Mundial del Comercio. (20 de noviembre de 2001). Declaración Ministerial de la omc (Doha, 2001). Recuperado el 25 de febrero de 2014 de omc: https://www.wto. org/spanish/thewto_s/minist_s/min01_s/ mindecl_s.htm

Rojas, S. (2006). Proyectos ambientales: una alternativa efectiva para las negociaciones sobre bienes y servicios ambientales en la омc.
En C. Correa (Ed.), Comercio internacional: del gatt a la omc. Disciplinas y solución de controversias (documento anexo). Buenos Aires: Editorial Eudeba.

Semana. (23 de junio de 2012). Río+20: palabras, tan solo palabras. Recuperado el 23 de febrero de 2014 de Semana: http://www. semana.com/nacion/articulo/rio20-palabras-tan-solo-palabras/259987-3

Semana. (20 de febrero de 2013). El mundo respira C02. Los diez países más contaminantes. Recuperado el 22 de febrero de 2014 de Semana: http://www.semana.com/especiales/contaminacion/index.html

Semana sostenible. (s.f.). Obama contra el calentamiento global. Recuperado el 15 de junio de 2014 de Semana: http://sostenibilidad.semana.com/medio-ambiente/articulo/el-plan-obama-contra-calentamientoglobal/31337

Ullah, F. (s. f.). A multi-stakeholder magazine outreach on climate change and sustainable development. Recuperado el 24 de febrero de 2014 de Stakeholder forum: http://www. stakeholderforum.org/sf/outreach/

Zelada, C. (2003). Comercio y medio ambiente: un asunto controvertido en la omc. Quito, Ecuador: Corporación Editora Nacional. 